\title{
Statistical Mechanics of a One-Dimensional Lattice Gas
}

\author{
D. RUELLE \\ I.H.E.S., 91. Bures-sur-Yvette \\ Received April 30, 1968
}

\begin{abstract}
We study the statistical mechanics of an infinite one-dimensional classical lattice gas. Extending a result of VAN Hove we show that, for a large class of interactions, such a system has no phase transition. The equilibrium state of the system is represented by a measure which is invariant under the effect of lattice translations. The dynamical system defined by this invariant measure is shown to be a $K$-system.
\end{abstract}

\section{Introduction and Statement of Results}

Let $\mathbb{Z}$ be the set of all integers $₹ 0$. We think of the elements of $\mathbb{Z}$ as the sites of a one-dimensional lattice, each site may be occupied by 0 or 1 particle. If $n$ particles are present on the lattice, at positions $i_{1}<\cdots<i_{n}$, we associate to them a "potential energy"

$$
U\left(\left\{i_{1}, \ldots, i_{n}\right\}\right)=\sum_{k \geq 1} \sum_{\left\{j_{1}, \ldots, j_{k}\right\} \subset\left\{i_{1}, \ldots, i_{n}\right\}} \Phi^{k}\left(j_{1}, \ldots, j_{k}\right) .
$$

The " $k$-body potential" $\Phi$ is a real function of its arguments $j_{1}<\cdots<j_{k}$ and is assumed to be translationally invariant i.e., if $l \in \mathbb{Z}$,

$$
\Phi^{k}\left(j_{1}+l, \ldots, j_{k}+l\right)=\Phi^{k}\left(\dot{j}_{1}, \ldots, j_{k}\right)
$$

Let $S \subset \mathbb{Z}$ and $K^{S}$ be the product of one copy of the set $K=\{0,1\}$ for each point of $S ; K^{S}$ is the space of all configurations of occupied and empty sites in $S ; K^{S}$ is compact for the product of the discrete topologies of the sets $\{0,1\}$. Let $\mathscr{C}\left(K^{S}\right)$ be the Banach space of real continuous functions on $K^{S}$ with the uniform norm and $\mathscr{M}\left(K^{S}\right)$ its dual, i.e. the space of real measures on $K^{S}$.

If $S \subset T \subset \mathbb{Z}$ we may write

$$
K^{T}=K^{S} \times K^{T \backslash S}
$$

and there is a canonical mapping $\alpha_{T S}: \mathscr{C}\left(K^{S}\right) \rightarrow \mathscr{C}\left(K^{T}\right)$ such that

$$
\alpha_{T S} \varphi\left(x_{S}, x_{T \backslash S}\right)=\varphi\left(x_{S}\right) .
$$

We denote by $\alpha_{S T}^{*}$ the adjoint of $\alpha_{T S}$ :

19 Commun. math. Phys., Vol. 9

$$
\alpha_{S T}^{*} \mu(\varphi)=\mu\left(\alpha_{T S} \varphi\right) .
$$


It will be convenient to use a functional notation for measures, writing $\mu(x) d x$ instead of $d \mu$. We have then

$$
\alpha_{S T}^{*} \mu\left(x_{S}\right)=\int d x_{T \backslash S} \mu\left(x_{S}, x_{T \backslash S}\right) \text {. }
$$

Let $(a, b]=\{i \in \mathbb{Z}: a<i \leqq b\}$ be a finite interval of $\mathbb{Z}$. The Gibbs measure $\gamma_{a b} \in \mathscr{H}\left(K^{(a, b]}\right)$ associates to each point $x=\left(x_{a+1}, \ldots, x_{b}\right)$ of $K^{(a, b]}$ the mass

where ${ }^{1}$

$$
\gamma_{a b}(x)=e^{-U(S(x))}
$$

$$
S(x)=\left\{i \in(a, b]: x_{i}=1\right\} .
$$

The measure $\gamma_{a b}$ is positive, has total mass

$$
Z_{b-a}=\int \gamma_{a b}(x) d x=\sum_{x_{a+1}=0}^{1} \cdots \sum_{x_{b}=0}^{1} \gamma_{a b}(x)
$$

and the corresponding normalized measure is

$$
\bar{\gamma}_{a b}=Z_{b-a}^{-1} \gamma_{a b} \text {. }
$$

Theorem 1. Let $\mathscr{E}$ be the space of sequences $\Phi=\left(\Phi^{k}\right)_{k \geqq 1}$ such that

if $\Phi \in \mathscr{E}$, then

$$
\sum_{l>0} \sum_{0<i_{1}<\cdots<i_{l}} i_{l}\left|\Phi^{\imath+1}\left(0, i_{1}, \ldots, i_{l}\right)\right|<+\infty
$$

(i) the following limit exists and is finite

$$
P(\Phi)=\lim _{b-a \rightarrow \infty} \frac{1}{b-a} \log Z_{b-a}
$$

it is continuously differentiable on any finte dimensional subspace of $\mathscr{E}$.

(ii) for every finite $S \subset \mathbb{Z}$ there exists $\varrho_{S} \in \mathscr{M}\left(K^{S}\right)$ such that

$$
\lim _{a \rightarrow-\infty, b \rightarrow \infty} \alpha_{S,(a, b]}^{*} \bar{y}_{a b}=\varrho_{S} .
$$

There is a measure $\varrho \in \mathscr{M}\left(K^{\mathbb{Z}}\right)$ such that

$$
\varrho_{S}=\alpha_{S \mathbb{Z}}^{*} \varrho
$$

for all finite $S \subset \mathbb{Z}$, and $\varrho$ depends continuously on $\Phi$ on any finite dimensional subspace of 6 for the vague topology of measures ${ }^{2}$.

This theorem expresses that a thermodynamic limit (infinite system limit) exists for the statistical mechanics of a one-dimensional lattice system if the condition (1.11) is satisfied. Furthermore the state of the infinite system, described by the measure $\varrho$, depends continuously on the temperature and chemical potential, which means that no phase transi-

${ }^{1}$ It is customary to write in $(1.7)$ instead of $U(S)$ the expression $\beta\left(-n \mu+U^{\prime}(S)\right)$ where $\beta^{-1}$ is the temperature, $\mu$ is the chemical potential and $U^{\prime}$ is computed by replacing $\sum_{k \geqq 1}$ by $\sum_{k>1}$ in (1.1). For notational convenience we absorb here $-\mu$ as $\Phi^{1}$ and $\beta$ as multiplicative constant in the definition of $U$.

2 I.e. the $w^{*}$-topology or the weak topology of $\mathscr{M}\left(K^{\mathbb{Z}}\right)$ in duality with $\mathscr{C}\left(K^{\mathbb{Z}}\right)$. 
tion can occur"; the system remains a "gas". If $\Phi^{l+1}=0$ for $l>1$, then (1.11) becomes

$$
\sum_{i>0} i\left|\Phi^{2}(0, i)\right|<+\infty
$$

This condition ensures that the energy of interaction of all particles at the left of a point of $\mathbb{Z}$ with all the particles at the right is bounded".

Given $S \subset \mathbb{Z}$, the translation $T^{l}: i \rightarrow i+l$ defines a homeomorphism of $K^{S}$ onto $K^{S+l}$ :

$$
T^{l}\left(\ldots, x_{-1}, x_{0}, x_{1}, \ldots\right)=\left(\ldots, x_{-l-1}, x_{-l}, x_{-l+1}, \ldots\right)
$$

and if $f \in \mathscr{C}\left(K^{S}\right), \mu \in \mathscr{M}\left(K^{S}\right)$ we define ${ }^{5} T^{l} f \in \mathscr{C}\left(K^{S+l}\right), T^{l} \mu \in \mathscr{M}\left(K^{S+l}\right)$ :

so that

$$
T^{l} f(x)=f\left(T^{-\imath} x\right), \quad T^{\imath} \mu(x)=\mu\left(T^{-\imath} x\right)
$$

$$
\mu\left(T^{l} f\right)=\int d x \mu(x) f\left(T^{-l} x\right)=\int d x \mu\left(T^{l} x\right) f(x)=T^{-\imath} \mu(f)
$$

Since the measure $\varrho$ is visibly $T$-invariant in $\mathscr{M}\left(K^{\mathbb{Z}}\right)$, the triple $\left(K^{\mathbb{Z}}, \varrho, T\right)$ is a dynamical system ${ }^{6}$.

Theorem 2. The dynamical system $\left(K^{\mathbb{Z}}, \varrho, T\right)$ is a $K$-system.

This implies that the measure $\varrho$ is ergodic and satisfies a "cluster property" (see Sec. 2) as one expects for a gas.

\section{Proof of Theorems 1 and 2}

Let $\mathbb{N} *=\{i \in \mathbb{Z}: i>0\}$ and $K_{+}=K^{N^{*}}$. For every integer $m \geqq 0$ we may write

$$
K_{+}=K^{(0, m]} \times T^{m} K_{+} \cdot
$$

In particular if $x \in K_{+}$; then $(0, x) \in K_{+},(1, x) \in K_{+}$.

We let $F_{\Phi} \in \mathscr{C}\left(K_{+}\right)$be given by

$$
F_{\Phi}(x)=\exp \left[-\sum_{l \geqq 0} \sum_{0<i_{1}<\cdots<i_{l}} x_{i_{1}} \ldots x_{i_{l}} \Phi^{l+1}\left(0, i_{1}, \ldots, i_{l}\right)\right]
$$

where $x=\left(x_{1}, \ldots, x_{i}, \ldots\right) \in K_{+}, x_{i}=0$ or 1 for each $i>0$. The continuity of $F_{\Phi}$ on $K_{+}$is ensured by (1.11). A mapping $\mathscr{L}_{\Phi}$ of $\mathscr{C}\left(K_{+}\right)$into itself is defined by

$$
\mathscr{L}_{\Phi} f(x)=f(0, x)+F_{\Phi}(x) f(1, x)
$$

3 This result was known when $\Phi$ has finite range, i.e. when there exists $L<+\infty$ such that $\Phi^{l+1}\left(0, i_{1}, \ldots, i_{l}\right)=0$ for $i_{l}>L$ (hence for $l>L$ ). In that case $P(\Phi)$ is real analytic on finite dimensional subspaces of $\mathscr{E}$ (is this true also here ?). A generalization of this result exists to continuous systems with a "hard core", see van Hove [5].

${ }^{4}$ If $\Phi^{2} \leqq 0$ and (1.15) is violated, the existence of a phase transition has been conjectured by M. FisHer [2] and M. KAC (private communications). I am indebted to M. FISHER for correspondence on this point.

5 We let formally $d\left(T^{l} x\right)=d x$.

- The notions of dynamical systems and of $K$-system are discussed in ARNoLD and AveZ [I] and JACOBS [3]. 
its adjoint $\mathscr{L}_{\Phi}^{*}: \mathscr{M}\left(K_{+}\right) \rightarrow \mathscr{H}\left(K_{+}\right)$is given by

$$
\left\{\begin{array}{l}
\mathscr{L}_{\circledast}^{*} \mu(0, x)=\mu(x) \\
\mathscr{L}_{\Phi}^{*} \mu(1, x)=F_{\Phi} \mu(x) .
\end{array}\right.
$$

Theorem 3. (i) For every $\Phi \in \mathscr{E}$ there exist $\lambda_{\Phi}>0, h_{\Phi} \in \mathscr{C}\left(\boldsymbol{K}_{+}\right)$, $v_{\Phi} \in \mathscr{M}\left(K_{+}\right)$such that $h_{\Phi}>0, v_{\Phi} \geqq 0, v_{\Phi}(1)=v_{\Phi}\left(h_{\Phi}\right)=1$ and $^{7}$

$$
\begin{aligned}
& \mathscr{L}_{\Phi} h_{\Phi}=\lambda_{\Phi} h_{\Phi} \\
& \mathscr{L}_{\Phi}^{*} \nu_{\Phi}=\lambda_{\Phi} \nu_{\Phi} .
\end{aligned}
$$

(ii) If $f \in \mathscr{C}\left(K_{+}\right)$the following limit

$$
\lim _{n \rightarrow \infty}\left\|\lambda_{\Phi}^{-n} \mathscr{L}_{\Phi}^{n} f-\nu_{\Phi}(f) h_{\Phi}\right\|=0
$$

holds uniformly for $\Phi$ in a bounded subset of a finite dimensional subspace of $\mathscr{E}$.

(iii) If $\mu \in \mathscr{M}\left(K_{+}\right)$the following limit

$$
\lim _{n \rightarrow \infty} \lambda_{\Phi}^{-n} \mathscr{L}_{\Phi}^{* n} \mu=\mu\left(h_{\Phi}\right) v_{\Phi}
$$

holds for the vague topology of $\mathscr{M}\left(K_{+}\right)$.

(iv) On any finite dimensional subspace of $\mathscr{E}, \lambda_{\Phi}$ is continuously differentiable, $h_{\Phi}$ is continuous for the uniform topology of $\mathscr{C}\left(K_{+}\right), v_{\Phi}$ is continuous for the vague topology of $\mathscr{M}\left(K_{+}\right)$.

This theorem will be proved in Sec. 3., here we use it to establish the results announced in See. 1. For notational simplicity we shall often drop the index $\Phi$ from $F, \mathscr{L}, \mathscr{L}^{*}, \lambda, h, v$,

Lemma. Let us write

$$
L=\lambda^{-1} \mathscr{L}, \quad L^{*}=\lambda^{-1} \mathscr{L}^{*} .
$$

(i) If $\mu \in \mathscr{M}\left(K_{+}\right)$, then

$$
\sum_{n_{1}=0}^{1} \cdots \sum_{n_{l}=0}^{1} L^{* l} \mu\left(n_{1}, \ldots, n_{l}, x\right)=L^{l} 1(x) \cdot \mu(x) .
$$

(ii) If $f \in \mathscr{C}\left(K_{+}\right)$, then

$$
\nu \cdot \alpha_{N^{*}, N^{*}+l} T^{l} f=L^{* l}(\nu \cdot f) .
$$

7 For every finite $S \subset \mathbb{N} *$ let

$$
\lim _{m \rightarrow \infty} \alpha_{S,(0, m]}^{*} \bar{\gamma}_{0 m}=v_{S} .
$$

One can show that $v_{\Phi}$ defined by Theorem 3 (i) is such that

$$
\nu_{S}=\alpha_{S N^{*}}^{*} v \text {. }
$$

The measure $v_{\Phi}$ describes thus the state of a system occupying the semi-infinite interval $(0,+\infty)=\mathbb{N} *$. 
We prove (i) by induction on $l$ :

$$
\begin{aligned}
& \sum_{n_{1}} \cdots \sum_{n_{l+1}} L^{* l+1} \mu\left(n_{1}, \ldots, n_{l+1}, x\right) \\
= & \sum_{n_{l+1}} L^{l} 1\left(n_{l+1}, x\right) \cdot L^{*} \mu\left(n_{l+1}, x\right) \\
= & L^{l} 1(0, x) \cdot L^{*} \mu(0, x)+L^{l} 1(1, x) \cdot L^{*} \mu(1, x) \\
= & L^{l} 1(0, x) \cdot \lambda^{-1} \mu(x)+L^{l} 1(1, x) \cdot \lambda^{-1} F^{\prime}(x) \cdot \mu(x) \\
= & L^{l+1} 1(x) \cdot \mu(x) .
\end{aligned}
$$

To prove (ii) it suffices to apply repeatedly the following identity

$$
\begin{aligned}
& {\left[v \cdot \alpha_{N^{*}, \lambda^{*}+1} T f\right]\left(n_{1}, x\right)=v\left(n_{1}, x\right) \cdot f(x)=L^{*} v\left(n_{1}, x\right) \cdot f(x) } \\
= & \left\{\begin{array}{l}
\lambda^{-1} v(x) \\
\lambda^{-1} F^{\prime}(x) v(x)
\end{array}\right\} \cdot f(x)=\left[L^{*}(\nu \cdot f)\right]\left(n_{1}, x\right)
\end{aligned}
$$

Let $\delta \in \mathscr{M}\left(K_{+}\right)$be the unit mass at $x_{0}=(0, \ldots, 0, \ldots)$. It is readily checked that

By (1.6), (1.9) we have

$$
\gamma_{0 m}=\alpha_{f 0, m], \mathbb{N}^{*}}^{*} \mathscr{L}^{* m} \delta
$$

$$
Z_{m}=\int \mathscr{L}^{* m} \delta(x) d x=\mathscr{L}^{* m} \delta(\mathbf{1})=\delta\left(\mathscr{L}^{m} 1\right)
$$

and using (2.7),

$$
\lim _{b \rightarrow a \rightarrow \infty} \frac{Z_{b-a}}{\lambda^{b-a}}=\lim _{n \rightarrow \infty} \frac{\delta\left(\mathscr{L}^{n} 1\right)}{\lambda^{n}}=v(\mathbf{1}) \cdot \delta(h)=h\left(x_{0}\right)>0
$$

which implies ${ }^{8}(1.12)$ with $P(\Phi)=\log \lambda_{\Phi}$ and Theorem 1 (i) follows from Theorem 3 (iv).

We study now the limit (1.13) with $S=(0, m]$ (this is sufficient because we may by translation of $\mathbb{Z}$ map $S$ into $(0, m]$ for some $m)$. Let $f \in \mathscr{C}\left(K^{(0, m]}\right)$, using (2.14), (2.16), part (i) of the Lemma and parts (ii), (iii) of Theorem 3 we get

$$
\begin{aligned}
& \lim _{a \rightarrow-\infty, b \rightarrow \infty} \alpha_{(0, m],(a, b]}^{*} \bar{\gamma}_{a b}(f) \\
& =\lim _{l, n \rightarrow \infty} \alpha_{[0, m],(-l, m+n]}^{*} \bar{\gamma}_{-l, m+n}(f) \\
& =\lim _{l, n \rightarrow \infty} \alpha_{(l, l+m],(0, l+m+n]}^{*} \bar{\gamma}_{0, l+m+n}\left(T^{l} f\right) \\
& =\lim _{l, n \rightarrow \infty} Z_{l+m+n}^{-1} \alpha_{(l, l+m], \mathbb{N}^{*}}^{*} \mathscr{L}^{* l+m+n} \delta\left(T^{l} f\right) \\
& =h\left(x_{0}\right)^{-1} \lim _{l, n \rightarrow \infty} \sum_{n_{1}=0}^{1} \cdots \sum_{n_{l}=0}^{1} \int d x L^{* l+m+n} \delta\left(n_{1}, \ldots, n_{l}, x\right) \\
& \text { - } \alpha_{\mathbb{N}^{*},(0, m]} f(x) \\
& =h\left(x_{0}\right)^{-1} \lim _{l, n \rightarrow \infty} \int d x L^{l} 1(x) \cdot L^{* m+n} \delta(x) \cdot \alpha_{\mathbb{N}^{*},(0, m]} f(x) \\
& =h\left(x_{0}\right)^{-1} \int d x \nu(1) h(x) \cdot \delta(h) v(x) \cdot \alpha_{\mathbf{N}^{*},(0, m]} f(x) \\
& =\int d x h(x) \cdot \nu(x) \cdot \alpha_{\mathrm{N}^{*},(0, m]} f(x) .
\end{aligned}
$$

${ }^{8}$ Actually (2.16) is a much stronger statement than (1.12). 
This establishes the existence of the limit (1.13) and shows that the measure $\varrho$ defined by (1.14) satisfies

$$
\alpha_{\mathbb{N}^{*} \mathbb{Z}}^{*} \varrho=h \cdot v .
$$

In view of Theorem 3 (iv), the r.h.s. of (2.17) is a continuous function of $\Phi$ on finite dimensional subspaces of $\mathscr{E}$. Because of the invariance of $\varrho$ under $T$, the same is true of $\varrho\left(\alpha_{\mathbb{Z} S} f\right)$ for every finite $S \subset \mathbb{Z}$ and $f \in \mathscr{C}\left(K^{s}\right)$. Part (ii) of Theorem 1 follows then from the density of

$$
\cup_{S} \alpha_{\mathbb{Z} S} \mathscr{C}\left(K^{S}\right)
$$

in $\mathscr{C}\left(K^{\mathbb{Z}}\right)$ for the uniform topology.

We come now to the study of the dynamical system $\left(K^{\mathbb{Z}}, \varrho, T\right)$. Let $\mathscr{B}_{1}$ be the algebra of all $\varrho$-measurable subsets of $K^{\mathbb{Z}}(\bmod .0)$ and $\mathscr{B}_{0}$ be the subalgebra consisting of the sets of measure 0 or 1 (i.e. $\emptyset$ and $K^{\mathbb{Z}}$ (mod. 0)). The system $\left(K^{\mathbb{Z}}, \varrho, T\right)$ is a $K$-system if there exists a subalgebra $\mathscr{A}$ of $\mathscr{B}_{1}$ such that

(i) $\mathscr{A} \subset T^{-1} \mathscr{A}$.

(ii) The union of the $T^{-\imath} \mathscr{A}$ generates $\mathscr{B}_{1}$.

(iii) The intersection of the $T^{l} \mathscr{A}$ is $\mathscr{B}_{0}$.

We write

$$
K^{\mathbb{Z}}=K^{S} \times K^{\mathbb{Z} \backslash S}
$$

and define $\mathscr{A}$ to be the subalgebra of $\mathscr{B}_{1}$ generated by all the sets $X \times K^{\mathbb{Z} \backslash S}$ where $X \subset K^{S}$ and $S$ is a finite subset of $N *$. The properties (i) and (ii) are then clearly satisfied. Let now $A \in \bigcap_{l \geq 0} T^{l} \mathscr{A}$ and $B$ be of the form $X \times K^{Z} \backslash S$ with $X \subset K^{S}, S$ finite $\subset \mathbb{N} *$. For all $l \geqq 0$ the characteristio function of $A$ may be written as $\alpha_{\mathrm{N}^{*}, \mathrm{~N}^{*}+1} T^{t} f_{l}$, let also $f_{B} \in \mathscr{C}\left(K_{4}\right)$ be the characteristic function of $B$. Using part (ii) of the Lemma, we get

$$
\begin{aligned}
\varrho(A \cap B) & =\int d x h(x) \cdot \nu(x) \cdot \alpha_{\mathbb{N}^{*}: \mathbb{N}^{*}+l} T^{l} f_{l}(x) \cdot f_{B}(x) \\
& =\int d x\left[L^{* l}\left(\nu \cdot f_{l}\right)\right](x) \cdot h(x) \cdot f_{B}(x) \\
& =\int d x \nu(x) \cdot f_{l}(x) \cdot\left[L^{l}\left(h \cdot f_{B}\right)\right](x) .
\end{aligned}
$$

Given $\varepsilon>0,(2.7)$ shows that, for sufficiently large $l$,

$$
\left\|L^{l}\left(h \cdot f_{B}\right)-v\left(h \cdot f_{B}\right) h\right\|<\varepsilon .
$$

From (2.20) and (2.21) we find

and therefore

$$
\begin{aligned}
\mid \varrho(A \cap B) & -\varrho(A) \varrho(B)|=| \int d x \nu(x) \cdot f_{l}(x) \cdot\left[L^{l}\left(h \cdot f_{B}\right)(x)\right. \\
& \left.-\nu\left(h \cdot f_{B}\right) h(x)\right] \mid<\varepsilon
\end{aligned}
$$

$$
\varrho(A \cap B)=\varrho(A) \varrho(B) .
$$

By translation, (2.23) remains true for any $B$ of the form $X \times K^{\mathbb{Z} \backslash S}$ with $X \subset K^{S}, S$ finite $\subset \mathbb{Z}$, and therefore for any $B \in \mathscr{B}_{1}$. In particular for 
$B=A$, we obtain $\varrho(A)=\varrho(A)^{2}$ hence $\varrho(A)=0$ or 1 , proving the property (iii) of $K$-systems and therefore Theorem 2.

Let $S$ be a finite subset of $\mathbb{Z}$ and define $f_{S} \in \mathscr{C}\left(K^{\mathbb{Z}}\right)$ by $f_{S}(x)=\mathbf{1}$ if $i \in S \Rightarrow x_{i}=1, f_{S}(x)=\mathbf{0}$ otherwise. The correlation function $\bar{\varrho}$ associated to $\varrho$ is a function of finite subsets of $\mathbb{Z}$ defined by

$$
\varrho(S)=\varrho\left(f_{S}\right) \text {. }
$$

Notice that by Theorem $1, \varrho_{\Phi}(S)$ is a continuous function of $\Phi$ on finite dimensional subspaces of $\mathscr{E}$. We have also

$$
\lim _{l \rightarrow \infty} \bar{\varrho}\left(S_{1} \cup T^{l} S_{2}\right)=\bar{\varrho}\left(S_{1}\right) \cdot \bar{\varrho}\left(S_{2}\right)
$$

a property known as cluster property and which should be possessed by the correlation function of a gas. The cluster property (2.25) is a consequence of strong mixing, which is a property of all $K$-systems ${ }^{9}$. The entropy of a $K$-system is $>0^{10}$, this entropy is identical to the mean entropy in the sense of statistical mechanics (see [4]). The $K$-system property (iii) has here a simple physical interpretation: it is not possible to make the system look different "at finite distances" by imposing restrictions "infinitely far away" on the configurations of the system (absence of long-range order).

\section{Proof of Theorem 3}

In this section we establish a series of propositions which will result in a proof of Theorem 3 .

For $m \geqq 0$ we let $\mathscr{C}_{m}=\alpha_{\mathbb{N}^{*},(0, m]} \mathscr{C}\left(K^{(0, m]}\right)$, i.e. $\mathscr{C}_{m}$ is the subspace of $\mathscr{C}\left(K_{+}\right)$consisting of those $f$ such that $f(x)=f\left(x^{\prime}\right)$ if $x_{i}=x_{i}^{\prime}$ for $i \leqq m$.

Proposition 1. Let $f \in \mathscr{C}_{m}, f \geqq 0$ and $x_{i}=x_{i}^{\prime}$ for $i=1, \ldots, k$. If $n \geqq 0, n \geqq m-k$, then

where

$$
A_{k}^{-1} \leqq \frac{\mathscr{L}^{n} f\left(x^{\prime}\right)}{\mathscr{L}^{n} f(x)} \leqq A_{k}
$$

$$
A_{k}=\exp \left[\sum_{l>0} \sum_{0<i_{1}<\cdots<i_{l}>k}\left(i_{l}-k\right)\left|\Phi^{l+1}\left(0, i_{1}, \ldots, i_{l}\right)\right|\right] .
$$

If $k \geqq m$, then $f\left(x^{\prime}\right)=f(x)$ and (3.1) holds thus for $n=0$. If $n>0$, (2.3) yields

$$
\frac{\mathscr{L}^{n} f\left(x^{\prime}\right)}{\mathscr{L}^{n} f(x)}=\frac{\mathscr{L}^{n-1} f\left(0, x^{\prime}\right)+F\left(x^{\prime}\right) \mathscr{L}^{n-1} f\left(1, x^{\prime}\right)}{\mathscr{L}^{n-1} f(0, x)+F(x) \mathscr{L}^{n-1} f(1, x)} .
$$

Using induction on $n$ we may assume that for $n_{1}=0,1$, we have

$$
A_{\bar{k}+1}^{-1} \leqq \frac{\mathscr{L}^{n-1} f\left(n_{1}, x^{\prime}\right)}{\mathscr{L}^{n-1} f\left(n_{1}, x\right)} \leqq A_{k+1}
$$

\footnotetext{
9 See [1] 11.4.
}

10 See $[1] 12.31$. 
and

$$
\begin{aligned}
& \exp \left[-\sum_{l>0} \sum_{0<i_{1}<\cdots<i_{l}>k}\left|\Phi^{l+1}\left(0, i_{1}, \ldots, i_{l}\right)\right|\right] \leqq \frac{F^{\prime}\left(x^{\prime}\right)}{F(x)} \\
\leqq & \exp \left[\sum_{l>0} \sum_{0<i_{1}<\cdots<i_{l}>l}\left|\Phi^{l+1}\left(0, i_{1}, \ldots, i_{l}\right)\right|\right] .
\end{aligned}
$$

Therefore

and (3.1) follows.

$$
\begin{aligned}
& A_{k}^{-1} \leqq \frac{\mathscr{L}^{n-1} f\left(0, x^{\prime}\right)}{\mathscr{L}^{n-1} f(0, x)} \leqq A_{k} \\
& A_{k}^{-1} \leqq \frac{F^{\prime}\left(x^{\prime}\right) \mathscr{L}^{n-1} f\left(0, x^{\prime}\right)}{F(x) \mathscr{L}^{n-1} f(0, x)} \leqq A_{k}
\end{aligned}
$$

Notice that if we write

$$
B=\exp \left[\sum_{l \geqq 0} \sum_{0<i_{1}<\cdots<i_{l}}\left|\Phi^{l+1}\left(0, i_{1}, \ldots, i_{l}\right)\right|\right]
$$

then $B^{-1} \leqq F(x) \leqq B$.

Proposition 2. There exist $v \in \mathscr{M}\left(K_{+}\right)$and $\lambda$ real such that $v \geqq 0$, $\|\boldsymbol{v}\|=1$ and

$$
\mathscr{L}^{*} v=\lambda \nu
$$

Furthermore $1+B^{-1} \leqq \lambda \leqq 1+B$ where $B$ is given by (3.8).

The set $\left\{\mu \in \mathscr{M}\left(K_{+}\right): \mu \geqq 0\right.$ and $\left.\mu(1)=1\right\}$ is convex, vaguely com. pact and mapped continuously into itself by

$$
\mu \rightarrow\left[\mathscr{L}^{*} \mu(1)\right]^{-1} \mathscr{L}^{*} \mu .
$$

By the theorem of SchaUder-Tychonov this mapping has a fixed point $\nu$ : (3.9) holds with $\lambda=\mathscr{L} * v(1)=v(\mathscr{L} 1)$. Since $\mathscr{L} 1(x)=1+F(x)$ and $B^{-1} \leqq F(x) \leqq B$, we have $1+B^{-1} \leqq \lambda \leqq 1+B$.

Proposition 3. (i) The closed hyperplane $H=\left\{f \in \mathscr{C}\left(K_{+}\right): v(f)=1\right\}$ is mapped into itself by $L=\lambda^{-1} \mathscr{L}$.

(ii) Let $f \in \mathscr{C}_{m}, f \geqq 0, n \geqq m$, then

$$
\begin{aligned}
& \sup _{x \in K_{+}} L^{n} f(x) \leqq A_{0} \nu(f) \\
& \inf _{x \in K_{+}} L^{n} f(x) \geqq A_{0}^{-1} v(f) .
\end{aligned}
$$

(iii) If $f \in \mathscr{C}\left(K_{+}\right)$, the sequence $\left\|L^{n} f\right\|$ is bounded by $A_{0}\|f\|$.

(iv) $A$ norm $\|\cdot \mid\|$ on $\mathscr{C}\left(K_{+}\right)$is defined by

$$
\||f|\|\left|=\nu(|f|)=\int d x \nu(x)\right| f(x) \mid \leqq\|f\| .
$$

(v) ||$L f f|\|\leqq\| f| \|$ for all $f \in \mathscr{C}\left(K_{+}\right)$.

(vi) If $f \in \mathscr{C}_{m}, v(f)=0$, and $n \geqq m$, then

(i) follows from

$$
\left\|\left|L^{n} f\right|\right\| \leqq\left(1-A_{0}^{-1}\right)\||f|\| .
$$

$$
v(L f)=\lambda^{-1} \mathscr{L}^{*} v(f)=v(f),
$$


(ii) follows from (3.1) with $k=0$ :

$$
\begin{aligned}
\nu(f)=\nu\left(L^{n} f\right) & \leqq \sup _{x^{\prime} \in K^{+}} L^{n} f\left(x^{\prime}\right) \\
& \leqq A_{\mathbf{0}} \inf _{x \in K_{+}} L^{n} f(x) \leqq A_{0} v\left(L^{n} f\right)=A_{0} \nu(f) .
\end{aligned}
$$

Using (3.11) with $m=0$ we have

which proves (iii).

$$
\left\|L^{n} f\right\| \leqq\left\|L^{n}|f|\right\| \leqq\|f\| \sup _{x \in K_{+}} L^{n} \mathbf{l}(x) \leqq A_{0}\|f\|
$$

It is clear that $\|\cdot\| \|$ is a semi-norm and that \|\|$f\|\|\|f\|$. We conclude the proof of (iv) by showing that if $f \geqq 0, f \neq 0$ then $\|\mid f\|>0$. We may indeed choose $m$ and $f^{\prime} \in \mathscr{C}_{m}$ such that $0 \leqq f^{\prime} \leqq t$ and $f^{\prime} \neq 0$, then $L^{m} f^{\prime}+0$ and (3.11) yields

$$
\|f\|\left\|=v(f) \geqq v\left(f^{\prime}\right) \geqq A_{0}^{-1}\right\| L^{m} f^{\prime} \|>0 .
$$

To prove $(\mathrm{v})$ we notice that

$$
\begin{aligned}
\||L f|\| & =v(|L f|)=\lambda^{-1} v(|\mathscr{L} f|) \leqq \lambda^{-1} v(\mathscr{L}|f|)=\lambda^{-1} \mathscr{L}^{*} v(|f|) \\
& =v(|f|)=\||f|\| .
\end{aligned}
$$

To prove (vi) let $f_{ \pm}=1 / 2(|f| \pm f)$, we have

$$
\left\|f_{+}\right\|=v\left(f_{+}\right)=v\left(f_{-}\right)=\left\|f_{-}\right\| .
$$

On the other hand by (3.12)

Therefore

$$
\inf _{x \in K_{+}} L^{n} f_{ \pm}(x) \geqq A_{0}^{-1}\|\| f_{ \pm}\|\|
$$

$$
\begin{aligned}
& \left\|\mid L^{n} f\right\|=v\left(\left|L^{n}\left(f_{+}-f_{-}\right)\right|\right) \\
& \left.=v\left(\left|L^{n} f_{+}-A_{0}^{-1}\right||| f_{+} \mid \|\right)-\left(L^{n} f_{-}-A_{0}^{-1}\|\| f_{-} \| \mid\right) \mid\right) \\
& \leqq \nu\left(\left|L^{n} f_{+}-A_{0}^{-1}\right||| f_{+}||||+\left|L^{n} f_{-}-A_{0}^{-1}\right||| f_{-}|| \mid\right) \\
& =\nu\left(L^{n}\left(f_{+}+f_{-}\right)-A_{0}^{-1}\left(\left\|\left|f _ { + } \left\|\left|+\left\|\left|f_{-} \|\right|\right)\right.\right.\right.\right.\right.\right. \\
& =\nu\left(L^{n}|f|-A_{0}^{-1}|\| f|||\right)=\nu(|f|)-A_{0}^{-1}|||f| \| \\
& =\left(1-A_{0}^{-1}\right)\|\| f \|
\end{aligned}
$$

which proves (3.14).

Proposition 4. Define

and

$$
\Sigma=\left\{f \in \mathscr{C}\left(K_{+}\right): v(f)=1, \quad f \geqq 0\right.
$$

$$
\left.A_{k}^{-1} \leqq \frac{f\left(x^{\prime}\right)}{f(x)} \leqq A_{k} \quad \text { if } \quad x_{i}^{\prime}=x_{i} \quad \text { for } \quad i=1, \ldots, k\right\}
$$


(i) $L \Sigma \subset \Sigma$.

(ii) If $f \in \Sigma$, then $\|f\| \leqq A_{0}$ and if $x_{i}=x_{i}^{\prime}$ for $i=1, \ldots, k$, then

$$
\left|f\left(x^{\prime}\right)-f(x)\right| \leqq A_{0}\left(A_{k}-1\right) \text {. }
$$

(iii) The set $\Sigma$ is convex and compact in $\mathscr{C}\left(K_{+}\right)$.

(iv) If $f, f^{\prime} \in \Sigma$, then

for all $k$.

$$
\left\|f-f^{\prime}\right\| \geqq B^{-k}(1+B)^{-k}\left(\left\|f-f^{\prime}\right\|-2 A_{0}\left(A_{k}-1\right)\right)
$$

(i) follows from Prop. 3 (i) and the same argument as in the proof of Prop. 1.

If $f \in \Sigma$, then $v(f)=1$ hence $v(f-1)=0$ and one can choose $\tilde{x}$ such that $f(\tilde{x}) \leqq 1$ hence $f(x) \leqq A_{0} f(\tilde{x}) \leqq A_{0}$, proving $\|f\| \leqq A_{0}$. If $x_{i}=x_{i}^{\prime}$ for $i=1, \ldots, k$ we get

$$
f\left(x^{\prime}\right)-f(x) \leqq f(x)\left(A_{k}-1\right) \leqq A_{0}\left(A_{k}-1\right)
$$

and (3.24) follows by exchanging the roles of $x$ and $x^{\prime}$.

The set $\Sigma$ is clearly convex and closed, since it is bounded and equicontinuous by (ii) the theorem of Ascons shows that it is compact, proving (iii).

Let $f, f^{\prime} \in \Sigma$. We can choose $\tilde{x}$ such that $\left|f(\tilde{x})-f^{\prime}(\tilde{x})\right|=\left\|f-f^{\prime}\right\|$. Denote by $g$ the characteristic function of the set $\left\{x \in K_{+}: x_{i}=\tilde{x}_{i}\right.$ for $i=1, \ldots, k\}$, using (ii) we obtain

$$
\left\|\left|f-f^{\prime}\right|\right\|=\nu\left(\left|f-f^{\prime}\right|\right) \geqq\left(\left\|f-f^{\prime}\right\|-2 A_{0}\left(A_{z^{2}}-1\right)\right) \cdot \nu(g)
$$

and (iv) follows from

$$
v(g)=\nu\left(L^{k} g\right)=\frac{\nu\left(\mathscr{L}^{k} g\right)}{\lambda^{k}} \geqq \frac{B^{-k}}{(1+B)^{k}},
$$

where we have used $F(x) \geqq B^{-1}, \lambda \leqq 1+B$ (see Prop. 2.).

Proposition 5. (i) There exists $h \in H$ such that $L h=h($ i.e. $\mathscr{L} h=\lambda h)$, $v(h)=1$.

(ii) If $f \in H$, then $\lim _{n \rightarrow \infty}\left\|L^{n} f-\not n\right\|=0$, more generally if $f \in \mathscr{C}\left(K_{+}\right)$, then

$$
\lim _{n \rightarrow \infty} L^{n} f=v(f) h
$$

in the uniform topology.

(iii) If $\mu \in \mathscr{M}\left(K_{+}\right)$the following limit exists in the vague topology

$$
\lim _{n \rightarrow \infty} \lambda^{-n}\left(\mathscr{L}^{*}\right)^{n_{n}} \mu=\mu(h) \cdot v .
$$

By Prop. 4 (i), (iii) the convex compact set $\Sigma$ is mapped into itself by $L$ which has therefore a fixed point $h$ by the theorem of SoraUdERTychonov, proving (i).

Let $f \in \Sigma$, in view of Prop. 4. (i), (ii), we can for each integer $n>0$ choose $m(n)$ independent of $N$ such that

$$
\left\|\left(L^{N} \tilde{f}-h\right)-g\right\|<\frac{1}{n !}
$$


for some $g \in \mathscr{C}_{m(n)}$ with $v(g)=0$. Then by Prop. 3. (v), (vi),

$$
\begin{aligned}
\left\|\mid\left(L^{N+m(n)} f-h\right)\right\| \| & \leqq\left\|L^{m(n)} g\right\| \|+\frac{1}{n !} \\
\leqq & \left(1-A_{0}^{-1}\right)\|g\|+\frac{1}{n !} \leqq\left(1-A_{0}^{-1}\right)\left\|L^{N} f-h\right\| \|+\frac{2}{n !} .
\end{aligned}
$$

If we put $M(n)=\sum_{i=1}^{n} m(i)$, we get

$$
\lim _{n \rightarrow \infty}\left\|\mid L^{N+M(n)} \tilde{f}-h\right\| \|=0
$$

uniformly in $N$, using then Prop. 4. (iv), we have thus

$$
\lim _{n \rightarrow \infty}\left\|L^{n} \tilde{f}-h\right\|=0
$$

when $f \in \Sigma$. This remains true if $f \in H$ and $f$ is a linear combination of elements of $\Sigma$, these linear combinations include the elements of $\mathscr{C}_{m}$ for all $m$ and are thus dense in $H$. By Prop. 3 (iii), $\left\|L^{n} f\right\|$ is bounded for all $f \in \mathscr{C}\left(K_{+}\right)$, hence the theorem of BanACH-STEINHAUs shows that

proving (ii).

$$
\lim _{n \rightarrow \infty}\left\|L^{n} f-y(f) \cdot h\right\|=0
$$

If $\mu \in \mathscr{M}\left(K_{+}\right)$, then for every $f \in \mathscr{C}\left(K_{+}\right)$

$$
\lim _{n \rightarrow \infty} \lambda^{-n}\left(\mathscr{L}^{*}\right)^{n} \mu(f)=\lim _{n \rightarrow \infty} \mu\left(L^{n} f\right)=\mu(\nu(f) \cdot h)=\mu(h) \nu(f)
$$

proving (iii).

Proposition 6. Let $\mathscr{F}$ be a finite dimensional subspace of $\mathscr{E}$ and $B$ a bounded subset of $\mathscr{F}$.

(i) The limit $\lim _{n \rightarrow \infty}\left\|L_{\Phi}^{n} f-\nu_{\Phi}(f) \cdot h_{\Phi}\right\|=0$ holds uniformly in $\Phi \in B$.

(ii) $h_{\Phi}$ is a continuous function of $\Phi \in \mathscr{F}$ for the uniform topology of $\mathscr{C}\left(K_{+}\right)$.

(iii) $v_{\Phi}$ is a continuous function of $\Phi \in \mathscr{F}$ for the vague topology of $\mathscr{M}\left(K_{+}\right)$.

(iv) Let $\Phi, \Psi \in \mathscr{F}, \Phi(t)=\Phi+t \Psi, t \in \mathbb{R}$, then the function $t \rightarrow \lambda_{\Phi(t)}$ has a derivative

$$
\frac{d}{d t} \lambda_{\Phi(t)}=v_{\Phi(t)}\left(\mathscr{L}_{\Phi(t), \Psi}^{\prime} h_{\Phi(t)}\right)
$$

where $\mathscr{L}_{\Phi, y}^{\prime}$ is the bounded operator on $\mathscr{C}\left(K_{+}\right)$defined by

$$
\begin{aligned}
\mathscr{L}_{\Phi, \Psi}^{\prime} f(x)= & {\left[-\sum_{l \geq 0} \sum_{0<i_{1}<\cdots<i_{l}} x_{i_{1}} \ldots x_{i_{l}} \Psi^{l+1}\left(0, i_{1}, \ldots, i_{l}\right)\right] } \\
& \cdot F_{\Phi}(x) f(1, x)
\end{aligned}
$$

and $\frac{d}{d t} \lambda_{\Phi(t)}$ is a continuous function of $\Phi \in \mathscr{F}$.

Let $f>0$ satisfy, for all $k$ and all $\Phi \in B$

$$
A_{\bar{k}}^{-1} \leqq \frac{\tilde{f}\left(x^{\prime}\right)}{\tilde{f}(x)} \leqq A_{k} \quad \text { if } \quad x_{i}^{\prime}=x_{i} \quad \text { for } \quad i=1, \ldots, k .
$$


Then, $v_{\Phi}(\tilde{f})^{-1} \tilde{f} \in \Sigma$. Since $A_{k}, B$ depend continuously on $\Phi \in \mathscr{F}$, the estimates in the proof of Prop. 5 (ii) can be made uniformly in $\Phi \in B$, hence

$$
\lim _{n \rightarrow \infty}\left\|v_{\Phi}(\tilde{f})^{-1} L_{\Phi}^{n} \tilde{f}-h_{\phi}\right\|=0
$$

uniformly in $\Phi \in B$. Since $v_{\Phi}(\tilde{f})<\|f\|$, (i) holds for $f=f>0$ satisfying (3.39).

In particular $L_{\Phi}^{n} 1$ tends to $h_{\Phi}$ uniformly in $\Phi \in B$, and $\| L_{\Phi}^{n} 1^{-1} L_{\Phi}^{n} 1$ $=\left\|\mathscr{L}_{\Phi}^{n} 1\right\|^{-1} \mathscr{L}_{\Phi}^{n} 1$, which is continuous in $\Phi \in B$, tends uniformly in $\Phi \in B$ towards $\left\|h_{\Phi}\right\|^{-1} h_{\Phi}$ which is therefore continuous in $\Phi \in \mathscr{F}$.

We have the identity

$$
t^{-1}\left(\lambda_{\Phi+t \Psi}-\lambda_{\Phi}\right) v_{\Phi}\left(\frac{h_{\Phi+t \Psi}}{\| h_{\Phi+t} w}\right)=v_{\Phi}\left(t^{-1}\left[\mathscr{L}_{\Phi+t \Psi}-\mathscr{L}_{\Phi}\right] \frac{h_{\Phi+t \Psi}}{\left\|h_{\Phi+t}\right\|}\right)
$$

and, in the norm of operators on $\mathscr{C}\left(K_{+}\right)$,

Therefore

$$
\lim _{t \rightarrow 0}\left\|t^{-1}\left(\mathscr{L}_{\Phi+t}-\mathscr{L}_{\Phi}\right)-\mathscr{L}_{\Phi, \Psi}^{\prime}\right\|=0 .
$$

$$
\lim _{t \rightarrow 0} t^{-1}\left(\lambda_{\Phi+t \Psi}-\lambda_{\Phi}\right)=\nu_{\Phi}\left(\mathscr{L}_{\Phi, \Psi}^{\prime} h_{\Phi}\right)
$$

which proves (3.37); $\lambda_{\Phi}$ is a continuous function of $\Phi \in \mathscr{F}$ because of the boundedness of $\left|v_{\Phi}\left(\mathscr{L}_{\Phi, \Psi}^{\prime} h_{\Phi}\right)\right|$ for $\Phi \in B$ (use $h \in \Sigma$ ).

We may consider $L^{n}: f \rightarrow L_{\Phi}^{n} f$ as a bounded operator from $\mathscr{C}\left(K_{+}\right)$to $\mathscr{C}\left(K_{+} \times B\right)$. For each $f \in \mathscr{C}\left(K_{+}\right)$the sequence $L_{\phi} f$ is bounded in $\mathscr{C}\left(K_{+} \times B\right)$ by Prop. 3 (iii). We have seen that (i) is satisfied for linear combinations of $f \geqq 0$ satisfying (3.39) for all $k$ and all $\Phi \in B$, these include again the elements of $\mathscr{C}_{m}$ for all $m$ and are thus dense in $\mathscr{C}\left(K_{+}\right)$. Applying the theorem of BANACH-STEINHAUs to the sequence $L^{n}$ proves then (i).

Applying (i) to $f=1$ yields (ii). More generally (i) shows that $v_{\Phi(f)} h_{\Phi}$ is continuous in $\Phi \in \mathscr{F}$, using then (ii) we see that $\boldsymbol{\nu}_{\Phi}(f)$ is continuous in $\Phi$ for each $f \in K_{+}$, proving (iii). Finally the continuity of the derivative (3.37) follows from the continuity in $\Phi \in \mathscr{F}$ of $v_{\Phi}$ (by (ii)), $h_{\Phi}$ (by (iii)) and $\mathscr{L}_{\Phi, \Psi}^{\prime}$.

\section{Bibliography}

1. Arnold, V. I., et A. Avez: Problèmes ergodiques de la mécanique classique. Paris: Gauthier-Villars 1967.

2. Frsher, M. E.: The theory of condensation (Sec. 6.). Lecture given at the Centennial Conference on Phase Transformation at the University of Kentucky, 1965.

3. JACOBS, K.: Lecture notes on ergodic theory. Aarhus Universitet (1962-1963).

4. Robinson, D., and D. Runlet: Mean entropy of states in classical statistical mechanics. Commun. Math. Phys. 5, 288-300 (1967).

5. VAN Hove, L.: L'intégrale de configuration pour les systèmes de particules à une dimension. Physica 16, $137-143$ (1950). 